\section{Reengineering (RE) TARMED}

\author{
W. Häuptli
}

Am Runden Tisch vom 30. April 2001 beschlossen die Tarifpartner im Beisein der Kantons- und Bundesbehörden eine Überarbeitung von TARMED, Version 1.0. Dabei wurde vereinbart, bis am 31. August 2001 mit einem Reengineering I schwergewichtig gefährdende Unwuchten im Tarif zu beheben und ein Konzept für das Reengineering II auszuarbeiten. Beides hat sich wegen einer zusätzlichen umfassenden redaktionellen Überarbeitung mit entsprechend hohem Aufwand für die informatische Umsetzung verzögert. So ist das Konzept RE II noch durch die Projektoberleitung zu genehmigen. Und die aus dem RE I hervorgegangene Version 1.1 von TARMED wurde erst vor kurzem publik.

In der Folge sollen die wichtigsten materiellen Änderungen des RE I aufgezeigt und diskutiert werden.

\section{Psychiatrie}

Wegen des Absentismus wurde die Produktivität der Sparte Sprechzimmerpsychiatrie auf 79\% gesenkt. Gemäss der Zeiterhebung entspricht dies einer $\mathrm{Ab}-$ senkung von 6\%. Zwar wird damit der Absentismus von rund 10\% nur teilweise kompensiert, weshalb zusätzlich die Limite bei Leistung in Abwesenheit des Patienten um 50\% erhöht wurde. Weiter wurde auf die Unterteilung in erste, weitere, letzte 5 Minuten im gesamten Kapitel 02 verzichtet. Schliesslich wurden für Krisenintervention und Testabklärung neue Positionen mit Limiten geschaffen, die für diese Situationen angemessen sind.

Damit wurde ein wesentlicher Teil der mangelhaften Abbildung der Arbeitsweise der Psychiater behoben und ihre einstige Forderung von Fr. 200.pro Stunde aus AL und TL erfüllt. Nicht eingetreten wurde auf den Burn-out, von dem Psychiater mehr als andere betroffen sein sollen.

\section{Pädiatrie}

Völlig überraschend deckte die Berechung des StartTPW eine ernsthafte Bedrohung dieser Fachgruppe auf. Als Grund fanden sich im Tarif nicht berück-

Korrespondenz:

Dr. med. Walter Häuptli

Schwanenplatz 7

CH-6004 Luzern sichtigte Unterschiede der Infrastruktur zwischen Allgemeinmediziner und Pädiater. Vor allem wird in der Allgemeinpraxis rund 8mal mehr geröntgt als beim Pädiater, womit in ersterer $8 \mathrm{mal}$ häufiger gleichzeitig die Personaldotation der Sparte Röntgen und beispielsweise der Sparte Sprechzimmer zur Verrechnung anfällt. Da die Zeit zur Behebung dieses Fehlers nicht reichte, erfolgte die Korrektur durch Kompensation auf der Gegenseite, nämlich einer Zuschlagsposition zur Grundkonsultation, erste $5 \mathrm{Mi}-$ nuten, verrechenbar von Pädiatern und Kinderchirurgen bei Untersuchung von Kindern unter 6 Jahren mit einer TL von 13 Taxpunkten.

\section{Dermatologie}

Ebenfalls als ernsthaft bedroht fanden sich die Dermatologen, Opfer der letzten 5 Minuten infolge ihrer gehäuften Kurzkonsultationen. Zur Abhilfe wurde ihnen ein Status gewährt mit dem zusätzlich wertvollen Nebeneffekt, dass sich auch diese Fachgruppe besser im Tarif erkennt.

\section{FMS}

Die Behandlung der Anliegen der FMS beanspruchte die meiste der ohnehin knappen Zeit des RE I. Deshalb mussten die Korrekturen einfach sein. Eine Hauptbeanstandung der FMS, nämlich die "verkehrte" und zu hohe Produktivität im OP, wurde korrigiert. Im OP I wurde sie von $72 \%$ auf $45 \%$ und im OP II und III von 65\% auf 50\% respektive 55\% gesenkt. Allerdings wurde ebenfalls der Faktor 1,2 aufgehoben, womit noch eine Verbesserung der AL um rund $40 \%$ im OP I und rund 20\% im OP II resultiert.

Die Dezimalen der Assistenz wurden aufgelöst. Bis 0,3 Assistenz wurden auf 0 (einiger weniger Leistungen auf 1), 0,4-1,3 auf 1 und über 1,3 auf 2 Assistenten gesetzt. Zusätzliche Assistenz ist neu bei einem Body Mass Index > 33 und unter speziellen Voraussetzungen bei kardialen Interventionen verrechenbar. Natürlich ist damit die Maximalforderung, nämlich der freien Bestimmung der Assistenz durch den Operateur, nicht gewährt. Durch Absenkung der Produktivität wurde aber immerhin die Forderung nach minimaler quant. Dignität FMH 5 der Assistenz bis auf sehr wenige Ausnahmen erfüllt.

Bezüglich der arztnahen TL, einer weiteren Forderung der FMS, wurde der Auflistung der darunter fallenden Elemente in den generellen Interpretationen zugestimmt. Abgelehnt wurde die quantitative Ausweisung, sei es in TP oder Prozent der TL, da dies von den Vertragsverhältnissen abhängt und diese unterschiedlich sind. Und keineswegs einverstanden waren die Versicherer mit der Subsumierung der arztnahen TL unter der AL, also der Vermischung von AL mit TL.

Schliesslich wurde die TL bei den Tarifpositionen für die Betreuung des hospitalisierten Patienten dem Besuch gleichgestellt, also beträchtlich erhöht. 
Insgesamt haben die Korrekturen eine markante Verbesserung der bisher entwürdigenden Situation bewirkt. Dem Wunsche nach Ersatz für die schwindende Zusatzversicherung konnte begreiflicherweise nicht entsprochen werden. Anderseits bestehen weitere berechtigte Forderungen der FMS, wie Sockelzeit und Schaffung neuer Positionen bei ausserordent- licher Beanspruchung. Doch diese Forderungen bedürfen umfassender und zum Teil bis aufs Einzelleistungsniveau reichender Abklärung und müssen deswegen im RE II aufgearbeitet werden. Sie sind notiert nebst anderem, das mindestens so vordringlicher Behandlung bedarf.

\section{Remaniement (RE) du TARMED}

\author{
W. Häuptli
}

Lors de la table ronde du 30 avril 2001, les partenaires tarifaires ont décidé, en présence des représentants des autorités cantonales et fédérales, de procéder à un remaniement (RE) de la version 1.0 du TARMED. Il a alors été convenu de remédier aux déséquilibres les plus graves présents dans le tarif (RE I) et d'élaborer un projet pour la deuxième phase de remaniement (RE II), le tout jusqu'au 31 août 2001. Or, en raison du toilettage rédactionnel qu'il a également fallu entreprendre et du temps nécessaire pour sa mise en application informatique, il n'a pas été possible de tenir ce délai. Ainsi, le projet RE II doit encore être approuvé par la direction générale du projet TARMED et le projet RE I vient seulement d'aboutir avec la publication de la version $1.1 \mathrm{du}$ TARMED.

Les principaux changements matériels apportés par le RE I sont exposés et discutés ci-après.

\section{Psychiatrie}

Le taux de productivité de l'unité fonctionnelle «salle de consultation psychiatrique» a été abaissé à 79\% en raison de l'absentéisme. D'après l'enquête sur le temps de travail, cela correspond à une diminution de 6\%. L'absentéisme, qui s'élève en tout à 10\%, n'est ainsi que partiellement compensé. Pour cette raison, on a également relevé de $50 \%$ la limite pour les prestations en l'absence du patient. De plus, on a renoncé,

Correspondance:

Dr Walter Häuptli

Schwanenplatz 7

CH-6004 Lucerne dans tout le chapitre, à la classification en: première période de $5 \mathrm{~min}$, par période de 5 min supplémentaire et dernière période de $5 \mathrm{~min}$. Enfin, pour les interventions de crise et les explications de tests, de nouvelles positions (avec limitation), adaptées à ces situations, ont été créées.

Il a ainsi largement été remédié à la prise en compte lacunaire des différents aspects du travail des psychiatres et leur ancienne exigence de Fr. 200.- par heure (prestation médicale [PM] et prestation technique [PT]) a été satisfaite. La question du «burn-out», qui semble affecter plus les psychiatres que d'autres confrères, n'a pas été abordée.

\section{Pédiatrie}

Contre toute attente, le calcul de la valeur initiale du point tarifaire a fait apparaître une grave menace pour ce groupement de spécialistes, provenant de la non-prise en compte dans le tarif de certaines différences d'infrastructure entre les généralistes et les pédiatres. On pratique en effet 8 fois plus de radiographies dans un cabinet de médecine générale que chez un pédiatre, si bien que, dans le premier cas, la dotation en personnel de l'unité radiologie et, par exemple, l'unité salle de consultation sont facturées 8 fois plus souvent. Comme le temps manquait pour remédier à cette erreur, on a opté pour une correction par compensation, en ajoutant notamment à la consultation, première période de $5 \mathrm{~min}$, une position additionnelle facturable par les pédiatres et les chirurgiens pédiatriques pour les enfants de moins de 6 ans (avec une PT de 13 points).

\section{Dermatologie}

Victime de la "dernière période de $5 \mathrm{~min}$ " du fait des très fréquentes consultations de courte durée, les dermatologues se sont, eux aussi, découverts sérieusement menacés. Pour les aider, un statut spécial leur a été accordé, si bien que ce groupement de spécialistes a également vu sa situation améliorée. 


\section{FMS}

Le traitement des demandes de la FMS a pris la majeure partie du temps - déjà limité - consacré au RE I. C'est pourquoi les corrections devaient être aussi simples que possible. L'une des erreurs principales soulignées par la FMS, à savoir la productivité trop élevée - confinant à l'absurdité - attribuée à la salle d'opération (OP), a été corrigée. Pour OP I, elle a été abaissée de $72 \%$ à $45 \%$, et pour OP II et III, de $65 \%$ à, respectivement, 50\% et 55\%. En outre, le facteur 1,2 a été supprimé, ce qui a permis d'obtenir en plus une amélioration de la PM de $40 \%$ pour OP I et de 20\% pour OP II.

Les décimales de l'assistance ont également été supprimées. L'assistance a été fixée à 0 (voire 1 pour de rares prestations) pour une valeur allant jusqu'à 0,3 , à 1 si la valeur se trouvait entre 0,4 et 1,3 , et à 2 pour une valeur supérieure à 1,3 . Une assistance supplémentaire peut également être facturée pour un indice de masse corporelle $>33$ et dans des conditions spéciales lors d'interventions cardiaques. Bien entendu, l'exigence maximale, à savoir la possibilité pour l'opérateur de fixer librement l'assistance, n'a pas été retenue. Quoi qu'il en soit, l'abaissement de la productivité a permis de tenir compte (à quelques exceptions près) de l'exigence d'une valeur intrinsèque quantitative minimale de FMH 5 pour l'assistance.
En ce qui concerne la $P T$ revenant au médecin, autre exigence de la FMS, la liste des éléments introduits à ce titre dans les interprétations générales a été acceptée. Par contre, la quantification en points tarifaires ou en pour-cent de la PT a été rejetée parce qu'elle dépend des rapports contractuels et que ceuxci peuvent différer. Par ailleurs, les assureurs rejettent l'idée d'adjoindre la PT médecin à la PM, autrement dit, ils refusent tout amalgame entre PT et PM.

Enfin, la PT pour les positions tarifaires de prise en charge $d u$ patient hospitalisé a été mise au niveau de celle pour la visite, c.-à-d. passablement rehaussée.

Dans l'ensemble, les corrections ont eu pour résultats une amélioration considérable de la situation, peu réjouissante jusqu'ici. Il n'a bien entendu pas été possible de répondre au souhait d'une compensation pour l'assurance complémentaire en déclin. Par ailleurs, d'autres prétentions justifiées de la FMS demeurent, telles qu'un temps de base et la création de nouvelles positions pour des contraintes exceptionnelles. Toutefois ces exigences demandent des clarifications approfondies, parfois même jusqu'au niveau de la prestation particulière, et seront donc abordées dans le cadre du RE II. Elles font partie d'une liste de points qui devront être traités en priorité. 